EUROPEAN ORGANIZATION FOR NUCLEAR RESEARCH

European Laboratory for Particle Physics

\title{
TOOLS FOR QUALITY TESTING OF BATCHES OF ARTIFACTS: THE CRYOGENIC THERMOMETERS FOR THE LHC
}

\author{
C. Balle ${ }^{1}$, J. Casas-Cubillos ${ }^{1}$, D. Ichim ${ }^{2}$ and F. Pavese ${ }^{2}$
}

\begin{abstract}
In the processing of data series, such as in the case of the resistance $\mathrm{R}$ vs. temperature $\mathrm{T}$ calibrations of the thermometers (several thousands) necessary for the LHC new accelerator at CERN, it is necessary to use automatic methods for determining the quality of the acquired data and the degree of uniformity of the thermometer characteristics, that are of the semiconducting type. In addition, it must be determined if the calibration uncertainties comply with the specifications in the wide temperature range 1,6-300 K. Advantage has been taken of the fact that these thermometers represent a population with limited variability, to apply a Least Squares Method with Fixed Effect. This allows to fit the data of all the thermometers together, by taking into account the individuality of each thermometer in the model as a deviation from one of them taken as reference

$$
\mathrm{R}_{i}=\mathrm{f}\left(T_{\mathrm{i}}\right)+b_{\mathrm{k} 0}+b_{k l} \mathrm{~g}\left(T_{k i}\right)+b_{k l} \mathrm{~g}\left(T_{k i}\right)^{2}+\ldots
$$

where $\mathrm{f}\left(T_{i}\right)$ is the model valid for all $\mathrm{i}$ data and all $k$ thermometers, while the subsequent part is the "fixed effect" model for the k-th thermometer, where $\mathrm{g}(T)$ is a suitable function of T. This method is shown in the paper applied to different stages of the data processing. First, for efficient compensation for the thermal drift occurring during acquisition, robust against the occurrence of outliers. Second, for detection of clusters of thermometers with inherently different characteristics. Finally, for optimisation of the calibration-point distribution.
\end{abstract}

1 CERN, LHC Division, Geneva, Switzerland

2 CNR, Istituto di Metrologia "G.Colonnetti", Torino, I taly

To be published in "Advanced Mathematical and Computational Tools in Metrology, V" Series on "Advances in Mathematics for Applied Sciences", World Scientific, Singapore, 2000

\footnotetext{
Administrative Secretariat

LHC Division

CERN

CH - 1211 Geneva 23

Switzerland

Geneva, 16 November 2000
} 


\title{
TOOLS FOR QUALITY TESTING OF BATCHES OF ARTIFACTS: THE CRYOGENIC THERMOMETERS FOR THE LHC
}

\author{
D.ICHIM, F.PAVESE \\ CNR, Istituto di Metrologia "G.Colonnetti”, Torino, Italy \\ E-mail:f.pavese@imgc.to.cnr.it \\ C.BALLE, J.CASAS-CUBILLOS \\ CERN, Geneva, Switzerland \\ E-mail: juan.casas-cubillos@cern.ch
}

\begin{abstract}
In the processing of data series, such as in the case of the resistance $R$ vs. temperature $T$ calibrations of the thermometers (several thousands) necessary for the LHC new accelerator at CERN, it is necessary to use automatic methods for determining the quality of the acquired data and the degree of uniformity of the thermometer characteristics, that are of the semiconducting type. In addition, it must be determined if the calibration uncertainties comply with the specifications in the wide temperature range $1,6-300 \mathrm{~K}$.

Advantage has been taken of the fact that these thermometers represent a population with limited variability, to apply a Least Squares Method with Fixed Effect. This allows to fit the data of all the thermometers together, by taking into account the individuality of each thermometer in the model as a deviation from one of them taken as reference:

$$
R_{i}=\mathrm{f}\left(T_{i}\right)+b_{k 0}+b_{k 1} \mathrm{~g}\left(T_{k i}\right)+b_{k 1} \mathrm{~g}\left(T_{k i}\right)^{2}+\ldots
$$

where $\mathrm{f}\left(T_{i}\right)$ is the model valid for all $i$ data and all $k$ thermometers, while the subsequent part is the "fixed effect" model for the $k$-th thermometer, where $\mathrm{g}(T)$ is a suitable function of $T$.

This method is shown in the paper applied to different stages of the data processing. First, for efficient compensation for the thermal drift occurring during acquisition, robust against the occurrence of outliers. Second, for detection of clusters of thermometers with inherently different characteristics. Finally, for optimisation of the calibration-point distribution.
\end{abstract}

\section{Introduction}

At the European Organization for Nuclear Research (CERN) a new accelerator of particles will be constructed, called Large Hadron Collider (LHC). For the construction of the LHC, a large amount of cryogenic thermometers has to be calibrated with automatic equipment and data reduction procedures in the temperature range $1,6 \mathrm{~K}-300 \mathrm{~K}$. The most important feature of the data set concerning the semiconducting-type thermometers, for which the specifications are stricter, is the limited uniformity of the dependent variable, i.e., the thermometer electrical resistance. We applied to this special kind of data a method, the Least Squares Method with Fixed Effect, that takes into account both the theoretical similarity of the thermometers and their individuality. [1,2] First, we studied an efficient compensation for the thermal drift occurring during acquisition, robust against the occurrence of outliers. Second, we used the method for detection of clusters of thermometers with inherently different 
characteristics. Finally, we used it for studying the optimisation of the calibrationpoint distribution.

\section{The Mathematical Model: LSM with Fixed Effect (LMSFE)}

The theoretical similarity of the thermometers imposed the choice of a model that assumes the same physical model for all the data series. However, a systematic bias between series must also be included. In the case of an additive bias characterizing each data series, the general description of the model is: $y_{k, i}=\operatorname{Common}\left(x_{k, i}\right)+\operatorname{Specific}\left(x_{k, i}\right)$, for $k=1, \ldots, S$ and $i=1, \ldots, n_{k}$ where $S$ is the total number of series and $n_{k}$ is the number of measurements of the $k$-th series, resulting in $T=\sum_{k=1}^{S} n_{k}$ total number of measurements. For simplicity, let us assume that there exists a polynomial basis for the function Common and that the specific effect is a linear one. Then the following model function holds:

$$
f(x ; j)=\sum_{h=0}^{m} a_{h} x^{h}+\sum_{k=1}^{S} d_{j, k}\left(t_{k}+u_{k} x\right) \text { where } \delta_{j, k}= \begin{cases}1 & \text { for } x \text { in the } k-\text { th series } \\ 0 & \text { otherwise }\end{cases}
$$

The $m+2 S+1$ parameters of the previous equation are not independent. Hence the parameters $a_{0}$ and $t_{k}$ are not separately estimable, and, similarly, the parameters $a_{1}$ and $u_{k}$. Constraining the least square estimates, the following model equation with $m+2 S-1$ independent parameters holds:

$f(x, j)=\left(a_{0}+t_{f}\right)+\left(a_{1}+u_{f}\right) x+\sum_{h=2}^{m} a_{k} x^{k}+\sum_{k=1}^{S} d_{j, k}\left[\left(t_{k}-t_{f}\right)+\left(u_{k}-u_{f}\right) x\right]$

where the $f$-th series is taken as reference.

Now the intercept $\delta=a_{0}+t_{f}$ of the reference series also represents the mean value of the $f$-th series, while each new bias parameter $\tau_{k}=t_{k}-t_{f}$ represents an effect relative to the reference. Similar considerations can be applied to the first derivative of the above equation for $x=0$, by defining $\gamma=a_{1}+u_{f}$ and $v_{k}=u_{k}-u_{f}$. Now, considering the $M$-dimensional vector $\alpha=\left(\delta, \gamma, a_{2}, \ldots, a_{m}, \tau_{2}, \ldots, \tau_{S}, v_{2}, \ldots v_{S}\right)$ and assuming $f=1$, the regression matrix becomes of the form $\left(X \mid \begin{array}{l}0 \\ D\end{array}\right)$, where $X$ is the $(T, m+1)$ classical regression matrix and $\left(T-n_{1}, 2(S-1)\right)$ matrix $D=\left(d_{i j}\right)$ is given by $d_{i j}= \begin{cases}1, & \text { if } j \leq S-1 \text { and } x_{i} \text { belongs to the } j+1-\text { th series } \\ x_{i}, & \text { if } S \leq j \leq 2 S-2 \text { and } x_{i} \text { belongs to the } j-S+2-\text { th series } \\ 0, & \text { otherwise }\end{cases}$

As usual, in this type of modeling procedures, a check on the scale of the variables is often needed In fact, in our studies we used, in general, a logarithmic 
transformation of the initial variables. Nevertheless, in this case the matrix $X$ could be ill-conditioned and a renormalisation should be performed.

\section{Applications and Results}

\subsection{Compensation of thermal drift}

Thermal drift with time in the calibration equipment is unavoidable, and there is advantage in using a method that allows for less thermal stabilisation, because it allows a net gain in the calibration time, provided that an internal check is possible for the effect of the thermal gradients.

In general, for drift compensation a reference calibrated thermometer is measured many times and its fitted data represent the drift function to be subtracted from the data. The LSMFE avoids the use of the reference thermometer, by fitting, on the contrary, the data of all the uncalibrated thermometers: few data points for each (typically 4-6), but on a large number of thermometers (15-50) allows an accurate evaluation of the drift function with a better statistics. The temperature span of the drift being limited, the thermometer individuality is limited too, and consequently a linear function Specific is generally sufficient.
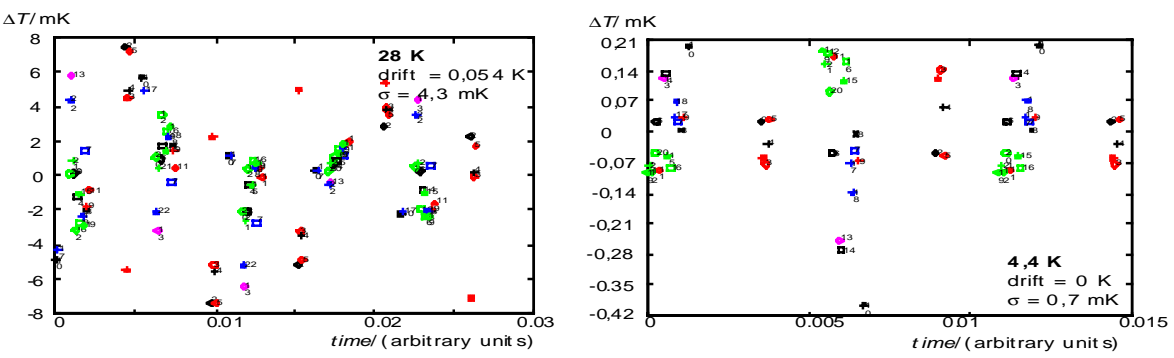

Fig. 1 - Reduction of the thermal drift with the LSMFE for 18 thermometers. Residuals of the overall fitting for very low thermal drift. Above $\approx 30 \mathrm{~K}$ the total drift increases up to tenths of a kelvin. However, the _values are always comparable with the ones estimated by using a reference thermometer, but they represent the actual uncertainty of the whole set of data.

The presence of outlying data required a preliminary screening procedure. These outliers can be due either to pulse-noise (for their elimination during data acquisition see [3]) or to outlying characteristics of a thermometer (either intrinsic or due to its mounting on the calibration apparatus). The outlier rejection has been simply obtained by applying a threshold criterium, not critical for the driftevaluation purpose. The quality of the resulting data series was still found to be quite variable, as shown in Fig. 1 for low-drift cases, reflecting the run-to-run variability of the thermal conditions in the calibration apparatus, though generally matching the tolerated uncertainty (see specs in Fig.3). Extending the LSMFE to 
larger and larger drift spans by adding data taken with higher drift rates, the dispersion of the data starts increasing in the high-drift region, detecting a new physical artifact, thermal gradients between thermometers. This can be used as a criterium for data selection.

\subsection{Fitting the $R$ - $T$ Characteristics}

The LSMFE was then applied to obtain the $R-T$ characteristics of the whole batch of thermometers. The polynomial $\log _{10}(T(R))=\sum_{i=0}^{m} A(i) \cdot\left(1 / \log _{10}(R)\right)^{i}$ was used with $i=$ 4 for the common model and with $i$ up to 2 for the fixed effect.

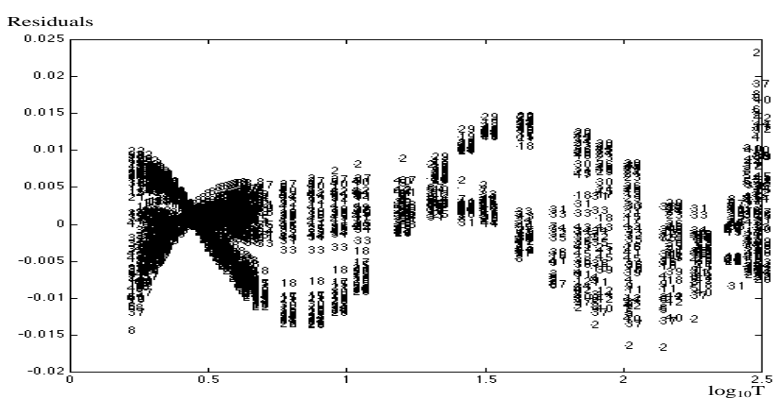

Fig.2 Clustering of 80 thermometers. Each cluster corresponds to a different set of model coefficients.

As shown in Fig.2, the LSMFE allows to detect groups of thermometers with characteristics intrinsically, though slightly, different. Especially with semiconducting thermometers, this could be correlated with fabrication parameters (position of the sensing elements on the chip) or with calibration parameters (mounting on the comparison block, overheating of the sensing element due to the measuring current). The latter has already been proved, while for the first a check will be done with the manufacturer.

For each group of homogeneous thermometers, the LSMFE has been applied a third time to check for the effect of the experimental design on the overall uncertainty. Fig. 3 shows the effect of the omission of all the experimental points in the temperature interval $77-300 \mathrm{~K}$ : the specs limits are not matched anymore. 


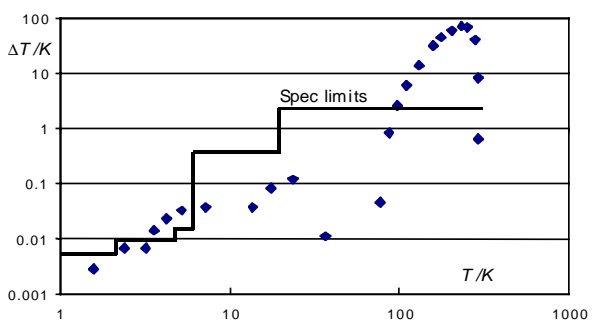

Fig.3. Deviation of the fitting when using a dataset with missing points between $77 \mathrm{~K}$ and $300 \mathrm{~K}$. The specs limits are also shown.

\section{Conclusions}

We have shown that the LSMFE can efficiently be used to compute thermal drift without resorting to a reference thermometer and being robust against mildly outlying data. We have also shown that the method is able to detect different classes of thermometers and to study the effect of experimental design on the whole batch of calibrations. A specific software running under MATLAB ${ }^{\circledR}$ is available for the purpose.

Studies are planned to continue on the minimisation of the calibration time, on the optimisation of the experimental design, also by using more stable classes of approximating functions (e.g., splines), and on the statistical evaluation of the results by using non-parametric methods (e.g., bootstrap).

\section{References}

1. Ciarlini P. and Pavese F., Application of special reduction procedures to metrological data, Numerical Algorithms, 5 (1993), pp. 479-489

2. Pavese F. and Cagna G., Thermal drift correction and precision evaluation by data processing of resistance thermometer comparisons, Inst. Phys. Conf. Ser, 26 (1975), pp. 117-124

3. Pavese F., Ichim D., Ciarlini P. and Regoliosi G., An algorithm for outlier rejection by sequence analysis in data acquisition: a first implementation for equispaced sequences, to appear in AMCTM $V$, World Scientific, Singapore, 2000

Work partially funded by CERN (90562GCH12Q121) 